EPJ manuscript No.

(will be inserted by the editor)

\title{
Violation of ensemble equivalence in the antiferromagnetic mean-field $X Y$ model
}

\author{
'Thierry Dauxois ${ }^{1 \mathrm{a}}$, Peter Holdsworth ${ }^{1}$ and Stefano Ruffo ${ }^{1,2 \mathrm{~b}}$ \\ 1 Laboratoire de Physique, UMR-CNRS 5672, ENS Lyon, 46 Allée d'Italie, 69364 Lyon Cédex 07, France \\ 2 Dipartimento di Energetica "S. Stecco", Università di Firenze, via S. Marta, 3, I-50139 Firenze, Italy
}

Received: date / Revised version: October 28, 2018

\begin{abstract}
It is well known that long-range interactions pose serious problems for the formulation of statistical mechanics. We show in this paper that ensemble equivalence is violated in a simple mean-field model of $N$ fully coupled classical rotators with repulsive interaction (antiferromagnetic XY model). While in the canonical ensemble the rotators are randomly dispersed over all angles, in the microcanonical ensemble a bi-cluster of rotators separated by angle $\pi$, forms in the low energy limit. We attribute this behavior to the extreme degeneracy of the ground state: only one harmonic mode is present, together with $N-1$ zero modes. We obtain empirically an analytical formula for the probability density function for the angle made by the rotator, which compares extremely well with numerical data and should become exact in the zero energy limit. At low energy, in the presence of the bi-cluster, an extensive amount of energy is located in the single harmonic mode, with the result that the energy temperature relation is modified. Although still linear, $T=\alpha U$, it has the slope $\alpha \approx 1.3$, instead of the canonical value $\alpha=2$.
\end{abstract}

PACS. 05.20.-y, Classical statistical mechanics - 05.20.Gg Classical ensemble theory - 05.45.-a Nonlinear dynamics and nonlinear dynamical systems

\section{Introduction}

The relation between microscopic dynamics and macroscopic thermodynamic behaviour can nowadays be explored in full detail in computer simulations [1]. This allows one to test important hypotheses of statistical mechanics. Formost among these is equivalence of ensembles.

It is widely accepted that the constant energy microcanonical ensemble gives the same results for average values as the constant temperature canonical ensemble. This is true under certain conditions [2], among them the most important is that interactions must be short-ranged. If interactions are long-range and attractive, as for gravitat' ing systems, all of thermodynamics breaks down due to the non extensivity of thermodynamic potentials [3]. For systems of Coulomb charges of opposite signs, screening effects may help in the construction of thermodynamics, but the problem is not trivially solved, even for classical systems [4], and each case must be examined separately.

In this context, mean-field models occupy a special status. Here, thermodynamic potentials can be made extensive if the thermodynamic limit is performed by rescaling the coupling with system size and letting the range of the interaction go to infinity [5]. Mean-field models are quite well studied in the canonical ensemble and serve as a

\footnotetext{
a Thierry.Dauxois@ens-lyon.fr

b INFM and INFN, Firenze (Italy)
}

zero-order characterization of phase transitions. Although their solution is often trivial for systems without disorder, they may hide important subtleties for disordered and frustrated systems [6]. Far fewer studies of mean-field models exist in the microcanonical ensemble. This may be the reason why, until now, ensemble equivalence has not been questioned for these models. In fact, one might have already expected some surprises on the basis of the exact solution of a model by Hertel and Thirring [7], where in the mean-field limit in the presence of extensive thermodynamic potentials ensemble inequivalence was explicitly shown.

In this paper we present a detailed study of the low temperature/energy phase of a model of classical rotators, whose potential energy is that of the mean-field antiferromagnetic XY model. The ground state of this model is highly degenerate, and while in the canonical ensemble equilibrium states are disordered at all temperatures (i.e. rotators do not display any directional organization), microcanonical ensemble simulations show the presence of a "bimodal" state where rotators are mainly grouped in two "clusters", pointing in directions separated by an angle $\pi$. This dynamical effect also has thermodynamical consequences: the order parameter measuring the degree of clustering is non-zero in the limit of zero energy and the energy temperature relation is not that predicted by the canonical ensemble. We find that, although the energy temperature relation $T=\alpha U$ is linear, as in the canonical 
case, the coefficient is $\alpha \approx 1.3$ and not the canonical value $\alpha=2$. From the point of view of dynamics, the rotators can be separated into two groups: a slow group which oscillates around the bi-cluster and a group of almost freely rotating rotators, which we call a "gas", following an analogy with particle motion.

In Section 2 we introduce the model. In Section 3 we discuss the unusual properties of its ground state and in the following Section 4 we present the main controversial points related to ensemble inequivalence. In Section 5 we present an analytical expression for the probability density function (PDF) for the orientation of a rotator, while the system is in the bi-cluster state, and we explain the consequences for the statistical properties in the system in the microcanonical ensemble. In Section 6 we discuss the main features of the dynamics of the moments of the PDF. The paper ends with some conclusions and perspectives.

\section{The model}

We consider classical rotators denoted by the angle $\theta_{i}$, $i=1, \ldots, N$, which all interact with each other, with an antiferromagnetic coupling $1 / N$ and an external field $h$

$$
V=\frac{1}{2 N} \sum_{i, j} \cos \left(\theta_{i}-\theta_{j}\right)-h \sum_{i} \sin \theta_{i}
$$

We will mostly restrict ourselves to $h=0$.

After defining the complex order parameters (with $i$ the imaginary unit)

$$
M_{k}=\frac{1}{N} \sum_{n} \exp \left(i k \theta_{n}\right)=\left|M_{k}\right| \exp \left(i \psi_{k}\right)
$$

the potential can be rewritten as

$$
V=N\left(\frac{\left|M_{1}\right|^{2}}{2}-h\left|M_{1}\right| \sin \psi_{1}\right)
$$

The microcanonical ensemble is obtained by adding a kinetic energy term to the above potential [8,9]

$$
H=K+V,
$$

with

$$
K=\sum_{n=1}^{N} \frac{p_{n}^{2}}{2} .
$$

In this formulation the model can also be thought of as describing a system of particles with unitary mass, interacting through the mean-field coupling $V$ (the names rotator and particle will be equivalently used throughout the paper). This model has been first introduced in Ref. [8] for $2 \mathrm{D}$ nearest-neighbour couplings and then studied in the antiferromagnetic mean-field context in Ref. [9].

The total energy $E=U N$ ( $U$ being the energy density) is fixed by the initial conditions and is conserved in time, while temperature is defined through the time averaged kinetic energy (see Ref. 10] for alternative definitions of temperature), $T=2<\bar{K}>/ N$, where $<\cdot>=$ $\lim _{t \rightarrow \infty} \frac{1}{t} \int_{0}^{t}$. If $h=0$, as is usually the case, then the total momentum $P=\sum_{n} p_{n}$ is also conserved. We set $P=0$ in order to avoid ballistic center of mass motion when $h=0$. The equations of motion

$$
\ddot{\theta}_{n}=\left|M_{1}\right|\left[\sin \left(\theta_{n}-\psi_{1}\right)+h \cos \theta_{n}\right]
$$

have been integrated using an improved fourth-order symplectic scheme [11]. The algorithm is $\mathcal{O}(N)$, provided one first computes $M_{1}$ in the central loop. During the time evolution, we sample the instantaneous values of the order parameters (2) up to $k=20$ and we compute their running time averages. System size was varied from $N=100$ to $N=10^{4}$. We have also performed canonical Monte-Carlo simulations, using the Metropolis algorithm, for comparison.

The initial conditions were of two classes: $i)$ homogeneous state $\theta_{n}=(2 \pi n) / N$, which can be shown to be marginally stable (see below), to which we add either a small spatially random perturbation $\theta_{n} \rightarrow \theta_{n}+r_{n}$ and/or a small momentum $p_{n}=r_{n}$ with zero average; ii) homogeneous state $\theta_{n}=(2 \pi n) / N$ with $p_{n}=A \sin \theta_{n}$, which leads to a faster induction of the bimodal state we want to study. These initial conditions lead to the same bimodal state, discussed below, at low energy.

The canonical solution of this model for $h=0$ is sketched in Ref. [9]. It uses the Hubbard-Stratonovich trick to decouple the rotators and the thermodynamic limit is performed by a saddle-point technique. The result is that all moments (2) vanish in the $N \rightarrow \infty$ limit, including the magnetization $M_{1}$, which implies, on the one hand, that

$$
T=2 U,
$$

on the other, that since in this limit

$$
M_{k}=\int_{0}^{2 \pi} \mathcal{P}(\theta) \exp (i k \theta) d \theta
$$

the $\operatorname{PDF} \mathcal{P}(\theta)$ is flat. Hence, the bimodal state is absent in the canonical ensemble, which we have confirmed by Monte-Carlo simulations.

Most of the discussions below will concentrate on the reasons for the different findings in the microcanonical and canonical statistical ensembles.

\section{Ground State and Statistical Properties in the Canonical Ensemble}

The long ranged interactions mean that it is impossible to satisfy all the antiferromagnetic bonds at once and the model is highly frustrated. In zero field, the frustration is minimized for configurations with $M_{1}=0$, giving a ground state energy of $U=E / N=0$. 
The ground state is infinitely degenerate, as there is an infinity of ways of minimizing the frustration. For example, grouping the rotators into pairs with angles $\theta_{i}$ and $\theta_{i}+\pi$ ensures that $M_{1}=0$ for all configurations of the pairs. The pairs do not have to be arranged in an ordered way however, as any disordered arrangement will equally give $M_{1}=0$. Neither is the ground state manifold restricted to pairs: one can equally construct ground states from groups of three, four, five ... rotators separated by angles of $2 \pi / 3,2 \pi / 4,2 \pi / 5 \ldots$. By moving rotators in clusters whose total angle is zero the system can evolve from one ground state to another remaining on the constant energy hypersurface.

The high dimensional ground state manifold follows from the fact that the ground state condition requires the two constraints $M_{1 x}=M_{1 y}=0$ only. One can therefore expect a ground state to possess $N-2$ unconstrained degrees of freedom, which is easily confirmed by calculating the Hessian $J_{i, j}=-\partial^{2} V / \partial \theta_{i} \partial \theta_{j}$. For example, for the perfectly homogeneous ground state at $h=0$, with $\theta_{i}=(2 \pi i) / N$

$$
J_{i, j}=-\frac{1}{2 N} \cos \left(\frac{2 \pi(i-j)}{N}\right) .
$$

The matrix indeed has two non-zero equal eigenvalues, $-1 / 4$ and $N-2$ zero eigenvalues.

Collective organization of the particles into reduced symmetry states can reduce the number of constraints even further. For example, in our system, if the rotational symmetry is broken and the spins lie along a single axis the ground state condition is reduced to a single constraint $M_{x}=0$, leading, at the harmonic level to $N-1$ free degrees of freedom [12. This result is confirmed by calculating the Hessian (9), on a perfect bi-cluster groundstate with $N / 2$ rotators at $\theta=0$ and $N / 2$ at $\theta=\pi$. One finds only one non-zero eigenvalue, $-1 / 2$, corresponding to the counter vibrating motion on the circle of the two groups of particles; all the other eigenvalues are zero.

The model we study is an extreme case of a collective paramagnet [13], or classical spin liquid [12,14]; a system that remains disordered with no evidence of spin freezing down to the limit of zero temperature. The special points on the ground state manifold with a reduced number of constraints can dominate the partition function, in the canonical ensemble, leading to an "Order by Disorder" transition [15] to a reduced symmetry state. However, the mode counting arguments of Moessner and Chalker 12. predict this to happen only if the number of liberated modes at the special points exceeds the number of zeromodes in a state with full symmetry. This is certainly not the case here and as we have confirmed by Monte Carlo simulation, Order by Disorder does not occur. We have simulated between $N=10$ and $N=10^{4}$ rotators down to temperatures $T<10^{-4}$. At all temperatures, the system remains perfectly disordered, with no evidence of bi-cluster formation. The number of zero modes can be directly verified by measuring the specific heat at constant field, $C_{h}$, at low temperature, as each quadratic mode makes a contribution $1 / 2$ (in units of $k_{B}$ ), while each zero mode makes a contribution zero. We find $C_{h}=1.0$, for the $N$ rotator system, as expected for two regular modes. There is therefore no evidence of the system preferring states with a single quadratic mode.

\section{Statistical Properties in the Microcanonical Ensemble}

The system reserves a surprise, when studied in the microcanonical ensemble, as we do not observe ensemble equivalence. For the classes of initial conditions cited above, the low energy state of the system is not one with a homogeneous distribution of angles, rather we observe the formation of a bimodal structure, with enhanced probability for two angles separated by a distance $\pi[9]$. As the energy goes to zero, the asymptotically reached state has broken symmetry, but is not perfectly bi-modal, as we discuss below. Nevertheless, when the Hessian for such a state is calculated numerically, we find that it also has only one non-zero eigenvalue. It seems therefore that the formation of the bicluster in the microcanonical ensemble, realises the condition of minimizing the number of non-zero modes.

After a transient time $\tau$, the formation of a stable bi-cluster is revealed by a non-zero value of the second moment $\left|M_{2}\right|$ of $\mathcal{P}(\theta)$. The early time evolution of $\left|M_{2}\right|$ shows the typical behavior observed for the growth of the mean-field in self-consistent dynamical models [16] with an initial exponential growth, followed by a saturation reached after a damped oscillatory motion has died away, see Figs. 1(a) and 1 (b). In Figs. 11(c) and 11(d) we show the space-time plot of $\mathcal{P}(\theta, t)$, which reveals the origin of the oscillations in the density waves originated periodically from the bi-cluster, preceding stabilization. The darkest colours correspond to the highest densities. A very sharply peaked but unstable bi-cluster forms over a short time period. The structure disperses with well defined instability edges that propagate from the cluster center and re-appears in a quasi-periodic manner with slowly lengthening period. The dispersing cluster appears to interact with propagating fronts from previous incarnations. The result is that the dispersion is successively slower and the fronts less well defined for following quasi-periods until finally the bi-cluster stabilizes. In addition Fig. 1 (d) shows that once this coherent structures has emerged and is stabilized, it propagates around the circle with apparently ballistic dynamics.

In Fig. 2(a), we show $\left|M_{2}\right|$, averaged over times later than $\tau$, as a function of temperature $T$, for various system sizes. For low temperatures $\left(T<10^{-4}\right),\left|M_{2}\right|$ approaches the value $\left|M_{2}\right| \sim 0.5$ and never decays, even in extremely long simulations (times as long as $10^{8}$ in proper unit: ${ }^{1}$ ). On the contrary, in the high temperature regime $\left(T>10^{-2}\right)$ the bi-cluster never forms, $\left|M_{2}\right| \sim 0$ for all times and the PDF remains flat, as in Monte-Carlo simulations. In the intermediate regime, $10^{-4}<T<10^{-2}$, the

\footnotetext{
1 The appropriate linear time scale of the system is $2 \pi=$ $\mathcal{O}(1)$
} 
Dauxois, Holdsworth and Ruffo: Violation of ensemble equivalence in the antiferromagnetic mean-field XY model
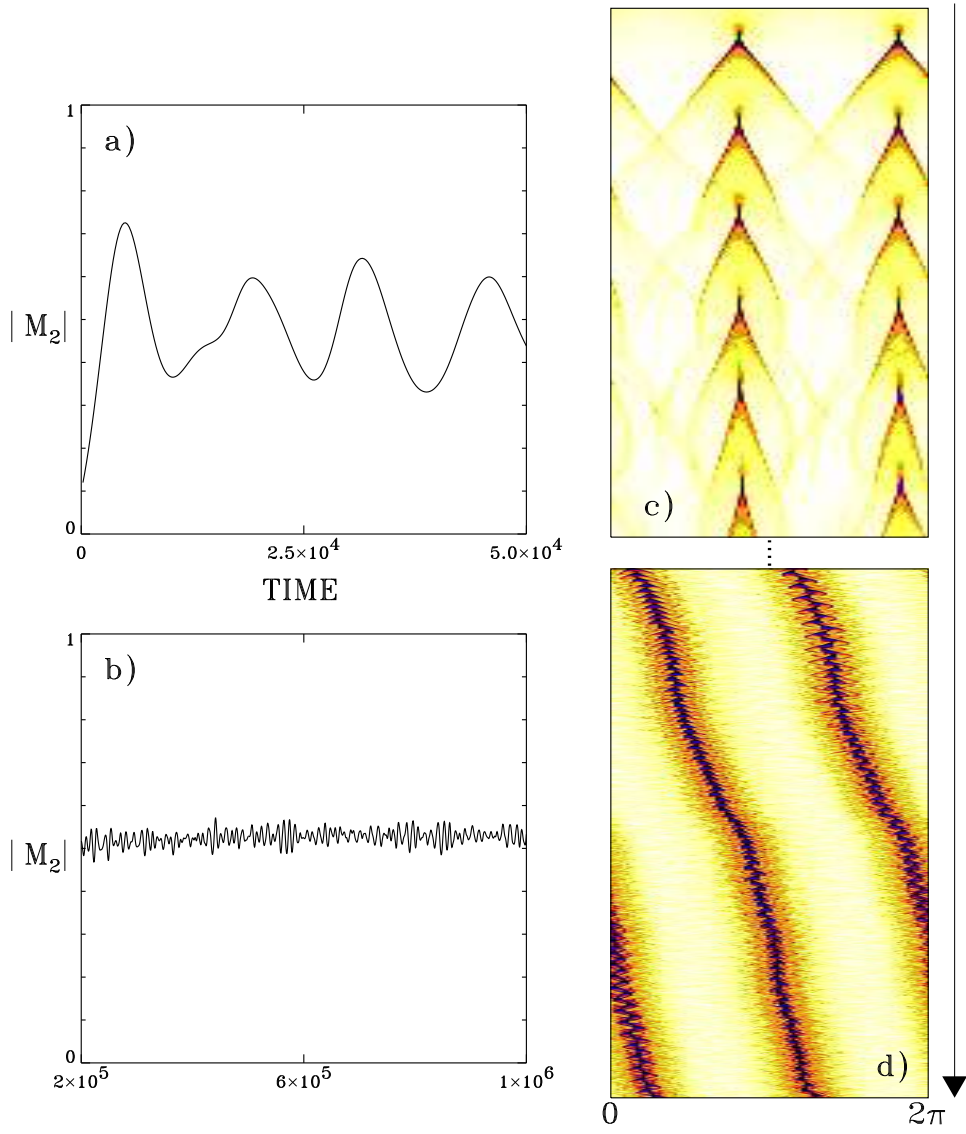

Fig. 1. (a) (resp. (b)) shows the instantaneous value of $\left|M_{2}\right|$ as a function of time at early (resp. later) times. (c) (resp. (d)) shows the evolution in grey scales of the distribution $\mathcal{P}(\theta, t)$ at early (resp. later) times. The $\theta$ axis is horizontal, the time axis vertical pointing downwards.

bi-cluster forms but is less well-defined, corresponding to progressively smaller values of $\left|M_{2}\right|$. As the temperature is increased there is a smooth transition to the homogeneous state observed in the canonical ensemble. In the next Section we will further discuss the internal structure of the bi-cluster.

The choice of initial conditions discussed above is dictated by the need to progressively increase the energy starting from a ground state. One may wonder what happens for more general initial conditions. We therefore looked at statistics of the final configuration, starting from many different realizations of a uniformly random distribution of angles and zero momenta. For $N=100$, at temperatures around $T \sim 10^{-5}-10^{-4}$, the bimodal state was always reached over 500 initial states. For $N=1000$ and $T \sim 10^{-6}$, the totality of 250 random initial states went to the bimodal state. It means that the bi-cluster is fully attractive for this class of initial states, which is quite generic.
As the formation of the bi-cluster is a unique property of the deterministic microcanonical system, it is an example of violation of the equivalence hypothesis between microcanonical and canonical behaviour. One might therefore expect this inequivalence to show up in other measurable quantities. This is indeed the case, for example we find that the standard relationship between energy and temperature is modified. In the canonical ensemble, as there are only two harmonic modes, equipartition of energy at low temperature leads to the energy temperature relation $E=N T / 2+T$ ( $N$ quadratic modes for the kinetic energy and 2 for the potential energy), which implies that $T=2 U+\mathcal{O}\left(N^{-1}\right)$, see (7). The eventual Order by Disorder averaging over parts of the phase space with only one quadratic modes (or any finite number of them) produces 舟ly $1 / N$ corrections to this relation. In the microcanonsimulation, in the presence of the bi-cluster we find the anomalous relation $T \sim 1.3 U$ as shown in Fig. 2(b). Once the temperature exceeds $T>10^{-2}$, the bi-cluster does not form and the energy-temperature relation crosses over to the canonical expression. In the presence of the bicluster, the kinetic energy is therefore much smaller than one should expect and the mean potential energy far in excess of that predicted by equipartition for the single non-zero mode associated with the bicluster. In fact, from this result, we find that the configurational contribution to the heat capacity $C_{h} \approx<V>/ T \sim(0.35 N) / 1.3$ is an extensive quantity, in complete contradiction to the predictions of mode counting in the canonical ensemble.

The system manages to put a macroscopic amount of energy in the single non-zero mode corresponding to the vibrating motion of the two groups of rotators and gives the system a low-dimensional aspect with the bi-cluster taking on many characteristics of a two-particle system. Fluctuations in the magnetization $\left|M_{1}\right|$, whose mean value is identically zero in the ground state illustrate this point. In zero field the potential energy is $V=N\left|M_{1}\right|^{2} / 2$, hence the extensive nature of the configurational heat capacity implies that mean value of the magnetization $\left\langle\left|M_{1}\right|>\right.$ should be of order unity and not of order $1 / \sqrt{N}$ as one might expect in an uncorrelated paramagnetic system. We have indeed observed ordered features in the time dependence of $M_{1}$ which are consistent with the nonfluctuational value of this quantity.

\section{Structural and Dynamical Details of the Bi-Cluster}

The bi-cluster never becomes perfectly formed, with $\left|M_{2}\right|=$ 1 , even in the limit $U \rightarrow 0$. Rather, the angular distribution of rotators retains a width, with a certain population of rotators homogeneously distributed. In Fig. 3 we show the angular $\operatorname{PDF} \mathcal{P}(\theta)$ for a system of $N=10^{4}$ particles and three different temperatures: one very low (Fig. $3(a)$ ) and two in the transition region (Fig. $3 \mathrm{~b}$ ) and (c)). The distribution is peaked for two angles, separated by $\pi$, but there remains non-zero probability of finding angles between the two peaks. The degradation of the bicluster 

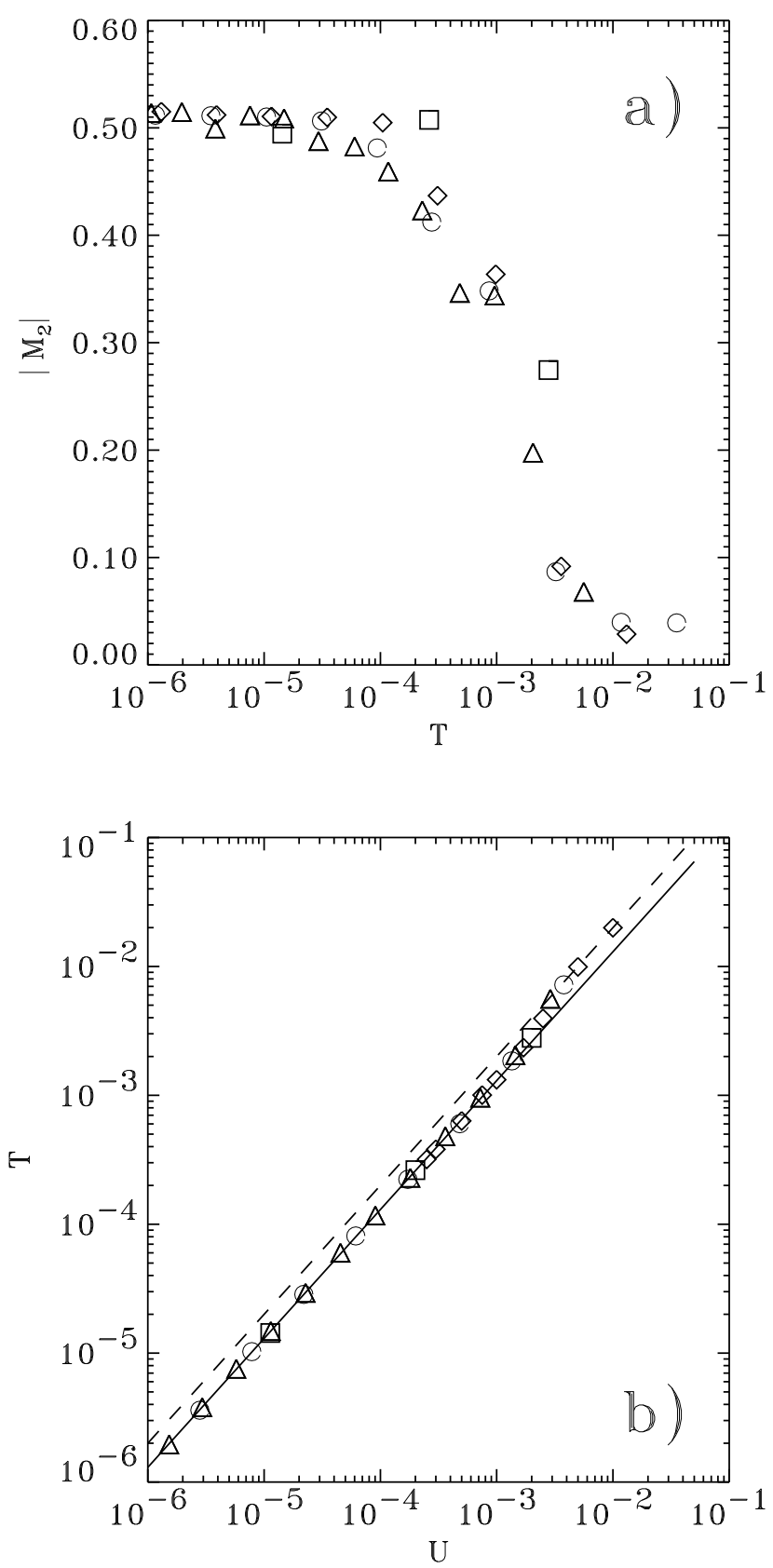

Fig. 2. (a) $\left|M_{2}\right|$ as a function of the temperature $T$ for $N=200$ (triangles), $N=500$ (circles), $N=10^{3}$ (diamonds), $N=10^{4}$ (squares). (b) $T$ vs. $U$ for the same values of $N$. The solid line is the modified microcanonical relation $T=1.3 \mathrm{U}$, whereas the dashed one corresponds to the canonical relation $T=2 U$ and is valid in the high energy regime, where the cluster has disappeared $\left(\left|M_{2}\right|=0\right)$. is progressive as the temperature is increased. We have made the experimental observation that the moments of the PDF assume the following values as the energy is decreased

$$
\begin{aligned}
& <\left|M_{k}\right|>\approx 0 \text { for odd } \mathrm{k} \\
& <\left|M_{k}\right|>=1 /|k| \text { for even } \mathrm{k} \text { with }<\left|M_{0}\right|>=1,
\end{aligned}
$$

as shown in the insets of Fig. 3 for the first $k=20$ modes. This observation offers no contradiction to our finding that $<\left|M_{1}\right|>$ is independent of system size. As the bi-cluster is stable at low temperatures only, the numerical value of $<\left|M_{1}\right|>$ remains small, even though it is an intensive quantity. By summing the Fourier series for $\mathcal{P}(\theta)$,

$$
\mathcal{P}(\theta)=\sum_{k=-\infty}^{\infty}<\left|M_{k}\right|>\exp (-i k \theta),
$$

one gets 17

$$
\mathcal{P}(\theta)=\frac{1}{2 \pi}(1-\log (2|\sin \theta|)) .
$$

This analytical formula is superimposed on to the numerical data in Fig. 3(a) with no free parameter apart from a shift of $\theta$, due to the motion of the bi-cluster (see below). The agreement is impressive, and although we have no theory for this result, we may well say we have a solution. We expect the analytical formula 12 to become exact in the $U \rightarrow 0$ limit.

The internal structure of the bi-cluster is further shown in Fig. 4(a), where the state at time $t=2.5 \cdot 10^{5}$, with $N=10^{4}$ and $T=1.43 \cdot 10^{-5}$, is displayed in the $(\theta, p)$ single-particle phase-space (so-called Boltzmann $\mu$-space). A large fraction of particles get stuck in the bi-cluster and have small values of $|\theta|$, while others develop much larger $\theta$ values. This illustrates why we speak of a "gascluster" coexistence: particles appear to belong to two distinct groups; those in the bi-cluster, which perform oscillations around the two centers separated by angle $\pi$, and those in the gas, which have ballistic dynamics and travel over large distances. Once projected onto the $p$-axis the distribution is symmetric (giving, for instance, zero average momentum), but as we see it in the $(\theta, p)$ space it is skewed, with, on average, bigger momenta for those particles which have traveled the furthest.

This picture is further clarified if we fold the $\theta$-axis onto $[0,2 \pi]$ (Fig. $4 \mathrm{~b})$. The oscillating motion of the particles of the cluster is now revealed by the spiral arms visible in the bi-cluster centers (see the inset). The particles in the cluster appear now to be distributed in a sinusoidal band of lesser density. Indeed, if we look inside the region around $\theta=0$ (Fig. 5a), we see that the particles are quite well distributed along the phase line

$$
p=A \sin (\theta+\phi),
$$

where $\phi$ is a sliding phase related to the bi-cluster motion and $A$ an energy related amplitude. This gives us a hint as to how to discover the dynamical mechanism at work 
Dauxois, Holdsworth and Ruffo: Violation of ensemble equivalence in the antiferromagnetic mean-field XY model
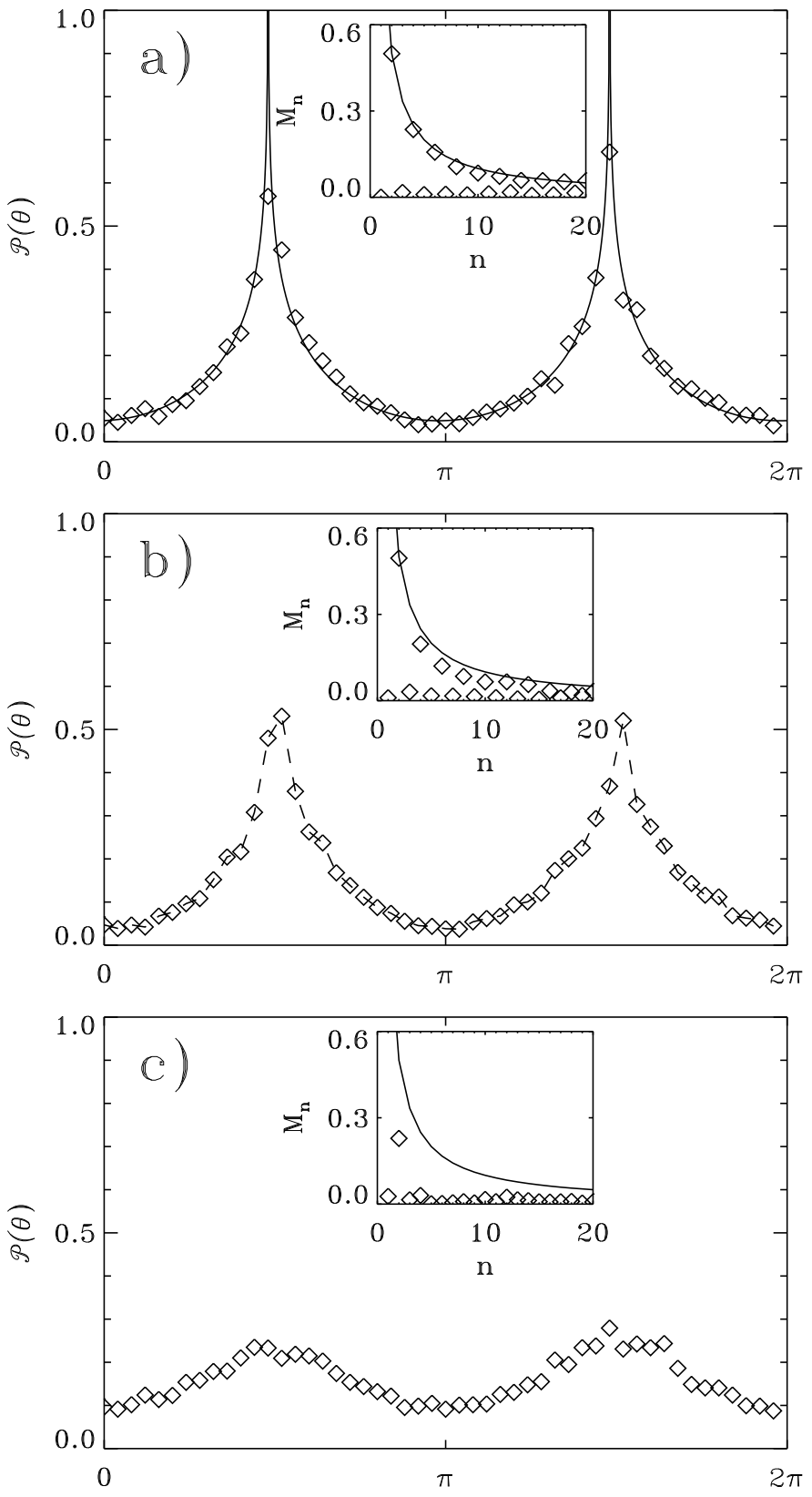

Fig. 3. $\mathcal{P}(\theta)$ for $N=10^{4}$ and three different values of $T$ : $1.46 \cdot 10^{-5}$ (a), $2.6 \cdot 10^{-4}(\mathrm{~b}), 2.8 \cdot 10^{-3}$ (c). The full line in (a) is the analytical formula (12), the diamonds are the numerical histograms (in (b) we join them for convenience with a dashed line). In the insets we show the corresponding momenta of the PDF (diamonds), the full line is $1 / n$. In (a) the moments respect the $1 / n$ law for even $n$, while in (c) only the mode $n=2$ is non-zero. Odd modes are much smaller.
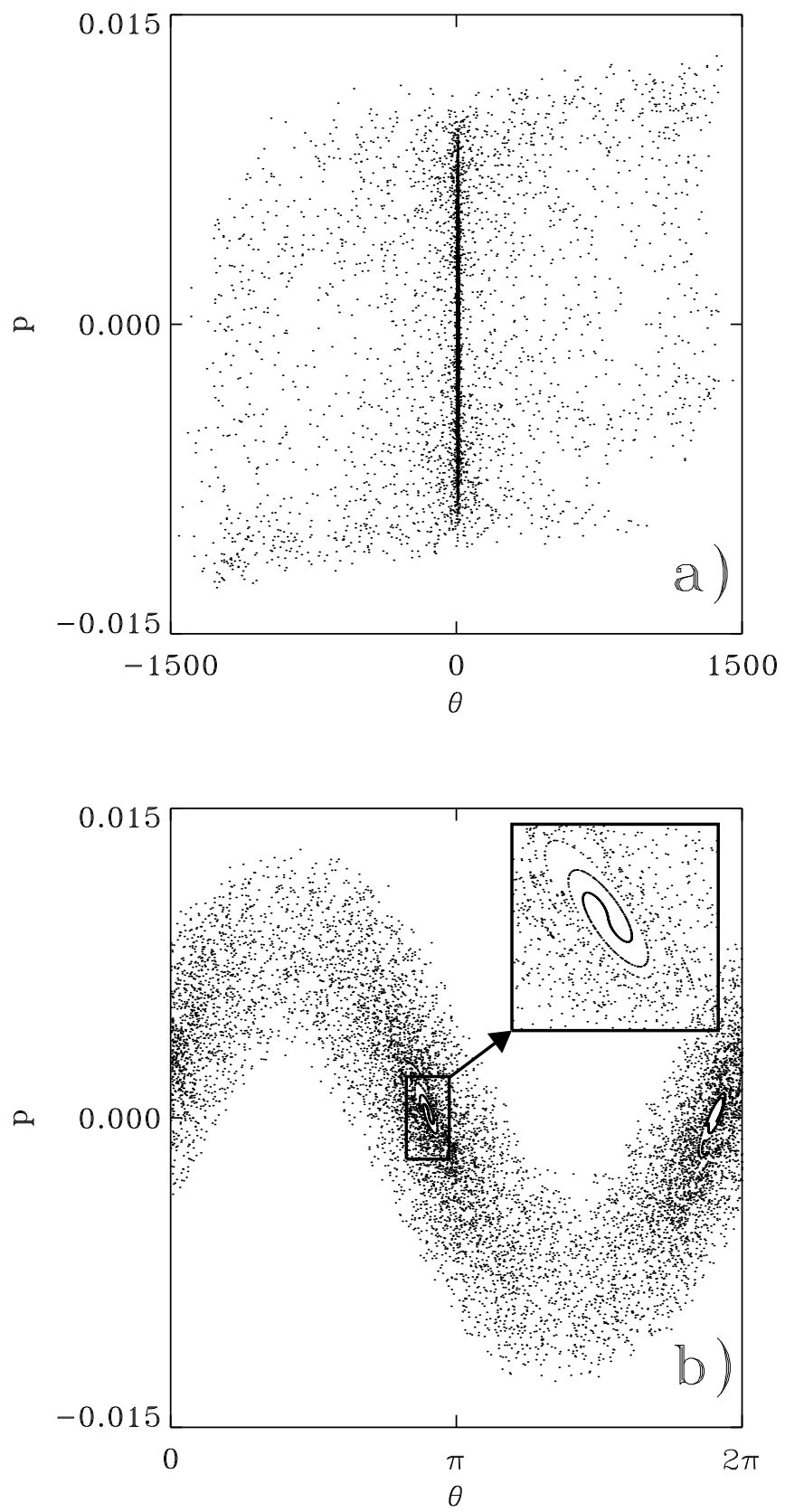

Fig. 4. Single particle phase-space for $N=10^{4}, U=1.13 \cdot 10^{-5}$ and $t=2.5 \cdot 10^{5}$. In (b) the angle $\theta$ is folded onto $[0,2 \pi[$ and the inset emphasizes the structure of the spirals arm around one of the bi-cluster center.

in the process of particle evaporation from the bi-cluster. In fact, differentiating (13) with respect to time and using $\dot{\theta}=p$, one gets for $u=2 \theta$ the equation of motion

$$
\ddot{u}=A^{2} \sin u,
$$

which describes a pendulum with gravity pointing upwards, $u=0$ being a saddle-point. This system is integrable and cannot produce the phase-distribution in Fig. 5(a). 
We must, therefore introduce some sort of perturbation capable of producing the sinusoidal layer observed in Fig. G(b). A well known mechanism for producing a stochastic layer in pendulum motion is the introduction of a finite time step, as done in the Chirikov standard map. We have therefore decided to iterate the map

$$
\begin{aligned}
u^{\prime} & =u+\Delta t p_{u} \\
p_{u}^{\prime} & =p_{u}+A^{2} \Delta t u^{\prime},
\end{aligned}
$$

with $\Delta t=1$, taking an ensemble of initial points homogeneously distributed in a small square around the unstable point $u=p_{u}=0$. A snapshot of these points at iteration 3000 is shown in Fig. 5(b); the particles have been stretched along the separatrix layer and distributed along it in a similar way to that shown in Fig. 5(a). Two features are responsible for the stretching: the unstable manifold distributes the particles away from the saddles, while the stable manifold attracts them to the saddles. The inhomogeneous distribution of the points is a result of these two mechanisms. One feature of the evaporation in the full model, which is not at all reproduced by the map (15), is the fact that time evolution seems to select only one of the two lobes of the separatrix; in other words, the symmetry $p \rightarrow-p$ seems to be broken in the full model, while stretching in both the lobes is present in the map (15).

This map is of course unable to explain why the bicluster forms and why it is stable. That is, if the evaporation mechanism were the only process present then the bi-cluster would be progressively depleted. This is in contrast with numerical simulations for low temperature values, which show the temporal stability of this collective state. Hence, a condensation mechanism on the bicluster should also exist, in order to establish a steadystate. Indeed, Fig. 1(b) shows that in the single-particle phase space two elliptic points are present at the centers of the bi-cluster, therefore in the bordering chaotic region of these two points a "trapping" effect could be present.

If formula (13) is approximately verified, then the knowledge of the PDF at low temperatures (12) would allow us to compute the relationship between the temperature and the constant $A$. Performing the integral, it turns out that

$$
T=<p^{2}>=\frac{A^{2}}{4} .
$$

This relation is very well verified numerically for low temperature values. Moreover, particle momenta appear to follow a distribution which, although non-Gaussian, has a variance given by $(16)$. The distribution shows a sharp peak in the center, due to the core of the bi-cluster, which is far from Gaussian. However, the wings of the distribution, which are due to the "gas" do follow a Gaussian distribution.

One might propose that total momentum conservation plays a role in the observed phenomenology. An easy way to test this idea is by adding a small external magnetic field $h$, which removes the constraint. We observe that, for small $h$, the bi-cluster still forms and is stable at low temperatures. The conservation law is not therefore relevant for cluster formation. On increasing $h$, the distribution $\mathcal{P}(\theta)$ is modified, as shown in Fig. 6. The bi-cluster
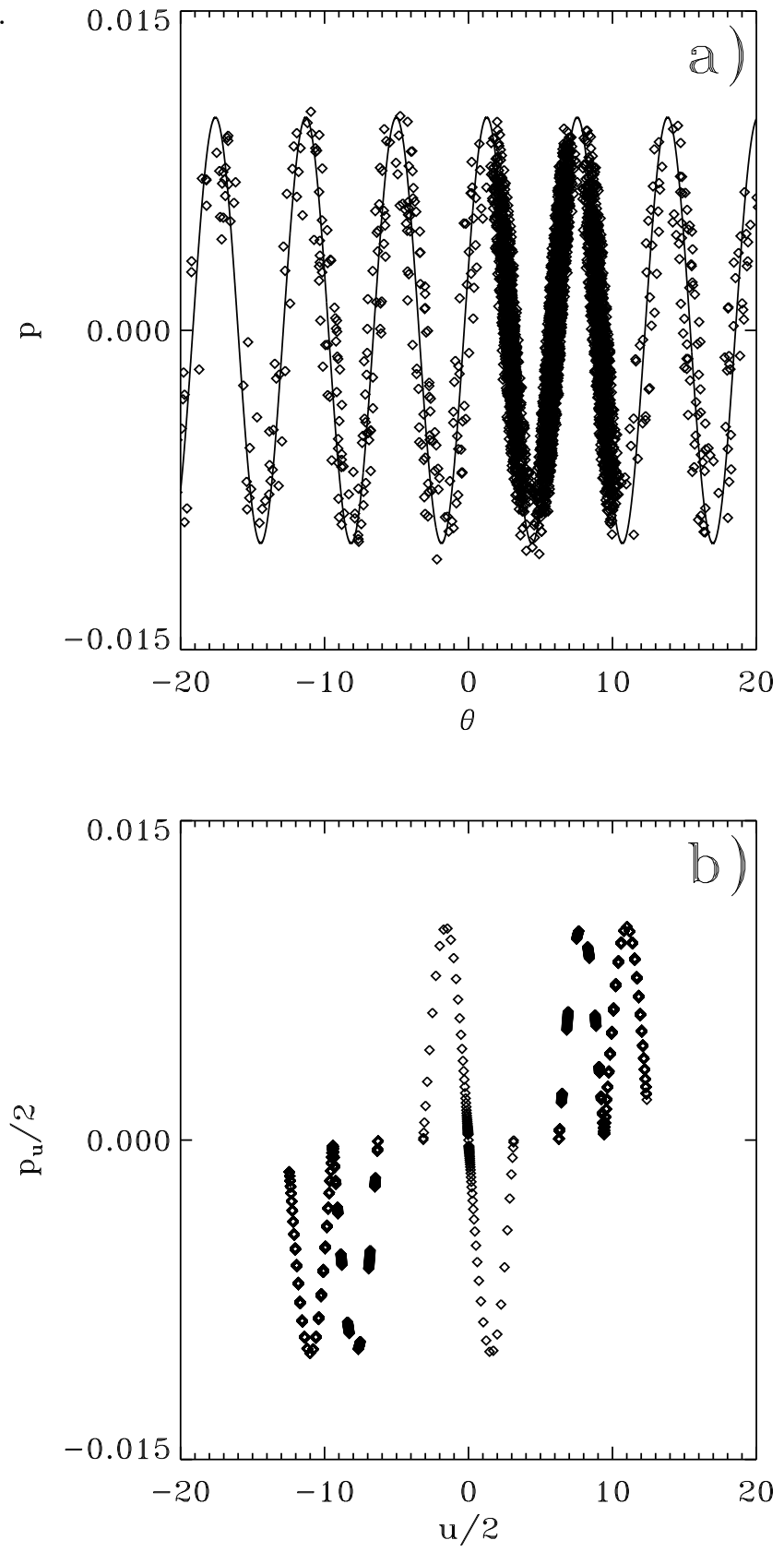

Fig. 5. (a) Zoom of the central region of Fig. Aa, showing the phenomenological law $p=A \sin (\theta+\phi)$ (13) with $A=0.01$ and $\phi=0.3$. (b) Phase-space of $10^{4}$ initial particles in the square $\left(u, p_{u}\right)=[-\pi * 0.0005, \pi * 0.0005]^{2}$ of the toy model $[15)$ (with $\Delta t=1)$ at iteration 3000 .

lies along the field axis and becomes asymmetric, with the number of rotators lying parallel and antiparallel to the field direction becoming unequal. The antiparallel cluster is continuously depleted until a single cluster eventually forms. This route towards a single cluster is somewhat counter intuitive if one thinks of a Néel ordered, unfrustrated antiferromagnet. Such a system would minimize its 


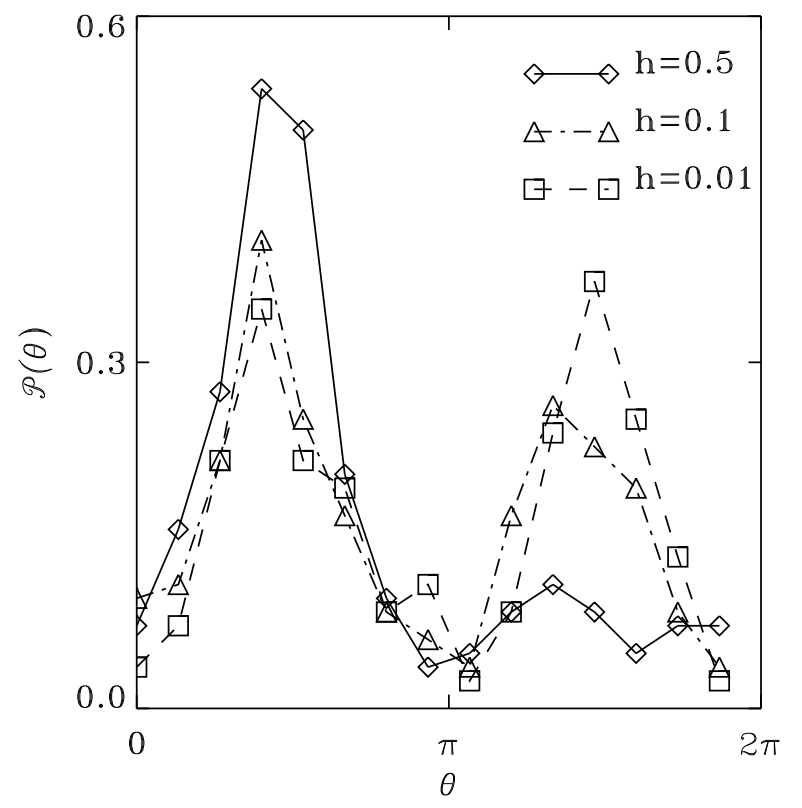

Fig. 6. $\mathcal{P}(\theta)$ in the presence of an external field for a chain of $N=200$ particles. The diamonds correspond to $h=0.5$ whereas the triangles to $h=0.1$ and the squares to $h=0.01$.

energy by aligning the bi-cluster perpendicular to the field and relaxing the two halves continuously out of the plane in a symmetric way. The bi-cluster would be destroyed by the two clusters, of equal size, rotating continuously onto the field direction.

\section{Moment dynamics}

As we have already remarked, there is no implicit contradiction between the proposed formula for the moments of the PDF (10) and the presence of a modified energy temperature relation. The latter means that $\left\langle\left|M_{1}^{2}\right|>\right.$ is an intensive quantity rather than being $\mathcal{O}(1 / N)$ as in the in the canonical ensemble. Hence, although all the other odd modes of the PDF vanish in the $N \rightarrow \infty$ limit, this is not true for $\left\langle\left|M_{1}\right|>\right.$. Rather, it remains finite in the low temperature regime, increasing linearly $U$. Solution (10) is therefore exact, only when $U \rightarrow 0$. However, because of the smallness of $U$ in this regime, $\left\langle\left|M_{1}\right|>\right.$ is a small quantity with respect to $\left\langle\left|M_{2}\right|>\right.$ and equation (10) is perfectly valid.

We expect that $M_{1}$ will display interesting dynamical behaviour, given its rather unexpected extensive nature, when the bi-cluster is formed. The $\left(\operatorname{Re}\left(M_{1}\right), \operatorname{Im}\left(M_{1}\right)\right)$ phase-plane is shown in Fig. 87(a) for $N=10^{4}$ and $U=$ $1.13 \cdot 10^{-5}\left(T=1.43 \cdot 10^{-5}\right)$. Successive points are joined by lines to show the relevant properties of the motion. The phase-point has a fast oscillatory motion through zero and a much slower rotatory motion centered on zero. The fast motion is due to the vibrations of the bi-cluster around the equilibrium positions (the two components of $M_{1}$ cross
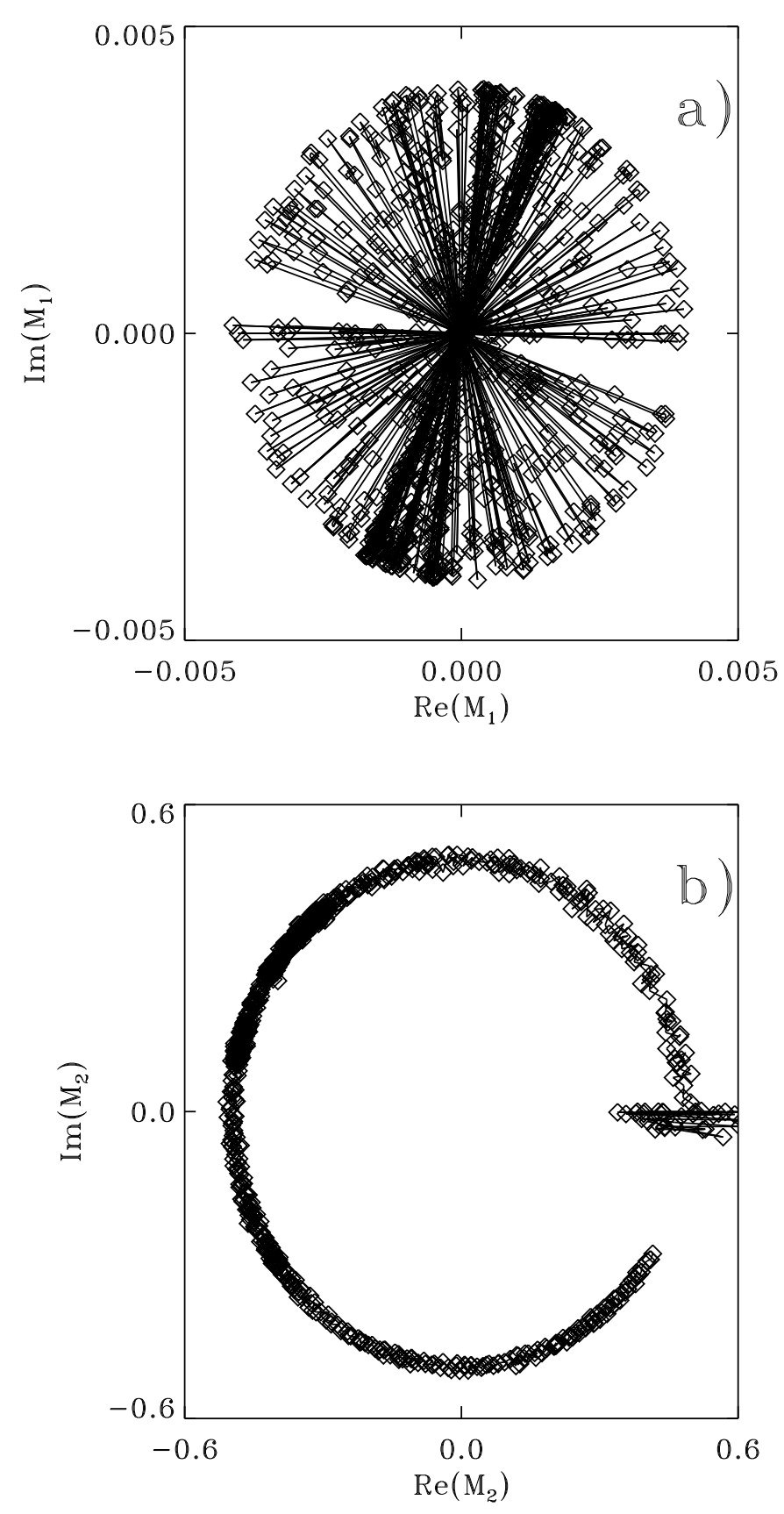

Fig. 7. (a) Phase-points of $M_{1}$ for $N=10^{4}$ particles and $U=1.13 \cdot 10^{-5}$. (b) Same for $M_{2}$.

zero in phase). The slow rotational motion is due to the rigid rotation of the bi-cluster. This latter motion is further revealed by the dynamics of $M_{2}$ in Fig. 7(b). Again we join successive phase-points with a line, showing that the phase point in the $M_{2}$ plane is rotating around the center, maintaining a fixed radius $\left|M_{2}\right| \approx 0.5$. The time dependence of the phase $\psi_{2}$ of $M_{2}$ is such that, over the long time span, the average is zero, but preliminary measurements of the variance $\sigma^{2}$ show that the motion is ballistic, rather than diffusive, $\sigma^{2} \sim t^{2}$. 
This picture is not only qualitative, but also quantitative. Indeed, if we average the fast oscillatory motion of $\left|M_{1}\right|$ in Fig. . (a), we get an estimate of $<\left|M_{1}\right|^{2}>$. Taking $<\left|M_{1}\right|^{2}>/ U \approx 0.7$, we get the correction to the energy temperature relation $T \approx(2-0.7) U=1.3 U$ as shown in Fig. 2(b).

At the transition temperature where $\left|M_{2}\right|$ is decreasing, both the motion of $M_{1}$ and that of $M_{2}$ become more erratic, revealing the beginning of the region where the bi-cluster is progressively depleting in time.

The other odd moments of the PDF always show an erratic motion around zero, with the variance of the cloud of points decreasing as $N$ is increased; i.e. higher odd modes are not intensive. The higher even moments of the PDF show a pattern similar to that of Fig. F(b), with a progressively reduced radius; the motion of the higher phases has also ballistic features.

\section{Conclusions and perspectives}

The antiferromagnetic mean-field classical rotator system is an ideal laboratory to study the relation between microcanonical and canonical ensembles. Although it has a trivial canonical solution, the randomly uniform state at all temperatures, the high degeneracy of its ground state induces nontrivial dynamical effects, which are revealed in the microcanonical ensemble. Instead of maintaining a random distribution of the rotators, the Hamiltonian dynamics selects a bimodal state, where the rotators are oriented along angles at distance $\pi$ with some spread, a bi-cluster. We have introduced an order parameter which reveals the formation of this state in the low energy phase and we have empirically obtained an analytical formula for the probability distribution function in angle, which perfectly fits the numerical data.

In addition to this first remarkable difference between the two ensembles, we have also shown that the energy temperature relation is modified and, although still linear, it has a different slope in the two ensembles. The origin of this behavior lies in the extensive amount of energy which Hamiltonian dynamics puts into the oscillatory vibrating motion of the bi-cluster.

Mean-field models are characterized by self-consistency. Indeed, a feature of our model is that rotators generate themselves the mean-field in which they move. Therefore, in the large $N$ limit, the dynamics of our model should depend only on the interaction of the single rotator with the mean-field. As already claimed for other models (e.g. beam-plasma instability and vorticity defect model [16]) self-consistency effectively reduces the number of degrees of freedom. This is why many properties of the dynamics of such a complex $N$-body system as ours resemble those of a "simple" perturbed pendulum. This is also the origin of the ordering of the rotators in a bi-cluster. There should be entropic reasons why the rotators prefer this state rather than choosing the disordered state, which is instead selected in the canonical ensemble.

Many questions remain to be explored, but the most compelling one concerns the careful description and expla- nation of the dynamics. The perturbed pendulum analogy must be further investigated and a thorough study of the time evolution of the moments of the probability distribution function in angle should allow a better understanding of the low-dimensional properties of the dynamics.

We thank M. Droz, J. Farago, M-C Firpo, M. Paliy and Z. Racz for useful discussions. S.R. thanks ENS-Lyon, INFN and INFM for financial support. P.H. thanks INFN for financial support. This work was performed using the computing resources of the Pôle Scientifique de Modélisation Numérique (PSMN) of ENS Lyon and of the DOCS-INFM group in Florence.

\section{References}

1. Chaos, Focus issue on chaos and irreversibility, T. Tèl, P. Gaspard and G. Nicolis Eds., (1998).

2. D. Ruelle, Statistical Mechanics: Rigorous Results, AddisonWesley, 1989.

3. T. Padmanabhan, Phys. Rep., 188, 285 (1990) and Refs. therein.

4. D.C. Brydges and Ph. A. Martin, J. Stat. Phys., 96, 1163 (1999) and Refs. therein.

5. M. Kac, G.E. Uhlenbeck and P.L. Hemmer, J. Math. Phys., 4, 216 (1963); ibidem, 5, 60 (1964).

6. M. Mezard, G. Parisi and M.A. Virasoro, Spin glass theory and beyond, World Scientific, Singapore (1987).

7. P. Hertel and W. Thirring, Ann. of Physics 63, 520 (1971).

8. J. Kogut and J. Polonyi, Nuclear Physics B265 [FS15], 313 (1986).

9. M. Antoni and S. Ruffo, Phys. Rev. E 52, 2361 (1995); K. Kaneko and T. Konishi, Physica D 71, 146 (1994); M. Antoni, Y. Elskens and C. Sandoz, Phys. Rev. E 57, 5347 (1998).

10. H.H. Rugh, Phys. Rev. Lett 78772 (1997); C. Giardiná and R. Livi, J. Stat. Phys. 91, 1027 (1998).

11. I. Mac-Laghlan and P. Atela, Nonlinearity 5, 541 (1992).

12. R. Moessner and J.T. Chalker, Phys. Rev. Lett. 80, 2929, (1998)

13. J. Villain, Z. Phys. B 33, 31, (1979).

14. J.T. Chalker, P.C.W. Holdsworth and E.F. Shender, Phys. Rev. Lett. 68, 855, (1992). J.N. Reimers and A.J. Berlinksy, Phys. Rev. B 48, 9539, (1993).

15. J. Villain, R. Bidaux, J.P. Carton and R. Coute, J. Phys. (Paris), 41, 1263, (1980).

16. J.L. Tennyson, J.D. Meiss and P.J. Morrison, Physica D 71, 1 (1994); D. del-Castillo-Negrete, Phys. Lett. A 241, 99 (1998).

17. I.S. Gradshtein and I.M. Ryzhik, Tables of Integrals, Series, and Products, Academic Press (1994), formula 1.441(2.). 